\title{
Role of Probiotic for Prevention and Management of COVID-19: A Literature Review
}

\author{
Asri Ludin Tambunan ${ }^{1}$, Gontar Alamsyah Siregar ${ }^{2 *}$ (D) \\ ${ }^{1}$ Department of Internal Medicine, Faculty of Medicine, Universitas Muhammadiyah Sumatera Utara, Medan, Indonesia; \\ ${ }^{2}$ Department of Internal Medicine, Faculty of Medicine, Universitas Sumatera Utara, Medan, Indonesia
}

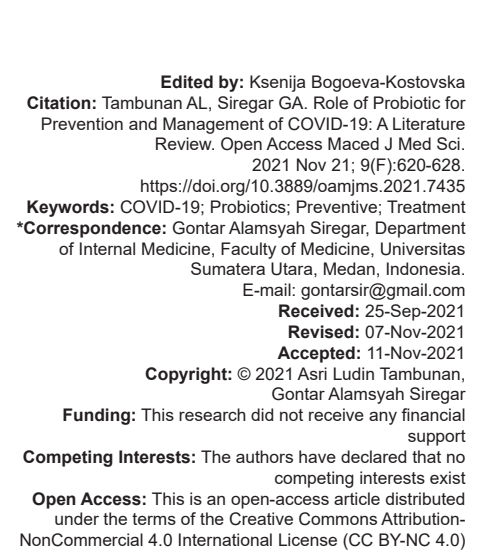

\section{Abstract}

BACKGROUNDS: Coronavirus (CoV) brought about by severe acute respiratory syndrome CoV 2 (SARS-CoV-2) is a continuous worldwide pandemic. SARS-CoV-2 influences the human respiratory tracts' epithelial cells, prompting a proinflammatory cytokine storm, and ongoing lung inflammation. With various patients kicking the bucket day by day, an immunization and explicit antiviral medication regimens are being investigated. The choice to utilize this medication during the COVID-19 pandemic should be founded on cautious thought of the likely as preventive and curative in such context.

METHODS: A literature review changed into carried out through the PubMed, ScienceDirect, Medline, and Google Scholar search engines consist of probiotics preventive and management possibility in COVID-19.

RESULTS: The probiotics significantly affects the host insusceptible reaction, foundationally, and on invulnerable responses at close by mucosal locales, such as the lung. Certain strains of probiotics have improved interferon type levels, hoisting the number and capacity of antigen-introducing cells, regular executioner cells, and T cells, just as expanding the degree of specific antibodies at the fundamental and mucosal destinations.

CONCLUSION: Probiotics have various advantages and potential in preventing and as the treatment of COVID-19, adjusting the arrangement of human gut microflora, reinforcing gut obstruction work, and defensive invulnerable reactions.

\section{Introduction}

Coronavirus (CoV) is another infection brought about by a novel COVID, severe acute respiratory syndrome CoV 2 (SARS-CoV-2), that essentially influences the lungs and aviation routes. While the total pathophysiology of the impact of this infection in man still needs to be characterized, it is apparent that its obsessive effects incorporate both direct impacts of viral intrusion and a complex immunological reaction. In reality, raised serum levels of the supportive of fiery cytokines have been accounted for to be prescient of a helpless guess in patients with COVID-19. Worldwide, there have been 197,134,265 affirmed cases of COVID19 in July 2021, including 4,210,148 passing, based on the World Health Organization data. There were $3,331,206$ cases with 90,552 deaths in Indonesia. The quantity of these cases proceeds to increment and changes each day. Episodes of infections and microorganisms starting from zoonoses in what's to come are probably going to proceed. Taking care of COVID-19 is at present at an unsuitable stage. There is no standard treatment for this infection and steady treatment is the lone procedure. Further, extended clinical preliminaries with better plans are as yet expected to assess the adequacy of this treatment albeit such medicines will be very difficult to embrace in a pandemic time [1], [2], [3]. COVID-19 not only affects global health system but also worldwide economy particularly regarding basic energy resources [4]. Prevention is an effective measure against COVID-19. By implementing social distancing (1-2 m), mask utilization, and sanitization, countries such as China and South Korea succeeded to suppress COVID-19 spread [5].

As 58-95\% COVID-19 inpatients have been put on empiric anti-infection agents to forestall ventilatorrelated pneumonia (VAP) and auxiliary contaminations, making an intrinsic test for antimicrobial stewardship programs. The support for the high utilization of empiric anti-toxins has been addressed dependent on the low pace of co-contaminations at confirmation (3-6\%), the low pace of auxiliary bacterial and parasitic diseases creating during hospitalization (4-14\%), and worries about the difficulties of the abuse of anti-infection agents. The patients conceded with COVID-19 keep on getting empiric anti-toxins (58-95\%), regardless of worries of anti-toxin abuse. This expanded utilization of anti-toxins has prompted concerns identifying with inconveniences related to anti-toxin use, including antibiotic-associated diarrhea (AAD) and Clostridioides difficile infections (CDI), the improvement of hypersensitivities or 
persistent fiery inside infection, and the potential for the advancement of anti-microbial safe bacterial strains [6].

Changed intestinal microbiomes have been identified in COVID-19 patients, which might make them more vulnerable to AAD or artful microorganisms such as Clostridioides difficile. Two potential techniques proposed to decrease anti-infection entanglements in COVID-19 patients have included anti-toxin stewardship projects to lessen the abuse of empiric anti-infection agents and the utilization of explicit probiotics to forestall AAD or CDI. Probiotics have been recently displayed to viably decrease $A A D$ and $C D I$ among different sorts of illnesses, because of numerous instruments of activity found in some probiotic strains, including the obliteration of pathogenic poisons, a deduction with microbe connection to have cells, and the capacity to go about as a resistant controller among different systems. One of the elective medicines accessible is Probiotics [7].

Probiotics are had faith in past examinations to have benefits in conquering medical issues. Probiotics are live microorganisms that can be found in aged food sources and refined milk and are known as "wellbeing amicable microscopic organisms," which show an assortment of useful wellbeing properties such as counteraction of intestinal illness, boosting the resistant framework, for lactose narrow mindedness and gut balance. Organisms, displaying against hypercholesterolemic and antihypertensive impacts, decreasing postmenopausal issues, and diminishing the runs. This proof gives off an impression of being satisfied concerning the counteraction and treatment of specific conditions while being possibly encouraging or even dubious concerning other people. Be that as it may, the improvement of probiotics for human utilization is as yet in its beginning phases [1].

Further exploration, as controlled human examinations, is expected to figure out which probiotics and portions are related with most prominent adequacy and for which patients, and to show their security and impediments. The fundamental focal point of Probiotics is on three things, well-being improvement, contamination control, and sickness the board using different sorts of direct utilization of Probiotics or with the utilization of food sources containing Probiotics. As of now, there isn't sufficient proof to suggest an explicit act against COVID-19 medicines. The choice to utilize this medication during the COVID-19 pandemic should be founded on cautious thought of the likely advantages and dangers for the patient [1], [6].

\section{Immune Responses in COVID-19}

$\mathrm{CoV}$ is wrapped and profoundly assorted RNA infections containing a huge (25-32 kb), single-abandoned, positive-sense RNA genome. There are four subfamilies of COVID, to be specific $\alpha$-; $\beta$-(beginning primarily from warm-blooded animals, especially from bats); gamma-and delta-(starting from pigs and birds). The $\alpha$ and $\beta$-COVIDS are known to cross creature human hindrances and cause extreme infection and fatalities. These COVIDS can taint people and just as a few different vertebrates and cause respiratory, enteric, hepatic, and neurologic infections. Up until now, six types of human COVIDS have been distinguished. Among them, SARS-CoV and the Middle East Respiratory Syndrome CoV (MERS-CoV) both having a zoonotic beginning, can cause extreme respiratory ailment with high mortality. Before 20022003, the SARS-CoV infection arose in China with an episode of SARS across 37 nations with $10.87 \%$ of causalities. Later in 2012, another respiratory diseaseMERS, brought about by MERS-CoV arisen with over $30 \%$ mortality in the Middle East nations. Until this point, three profoundly pathogenic and deadly COVIDS that taint people are SARS-CoV, MERS-CoV, and the as of late detailed one is SARS-CoV-2 [8], [9], [10], [11].

Autophagy is simply the cell's annihilation cycle. It is a cycle that starts with the endoplasmic reticulum (ER) under pressure and finishes with dangerous metabolites in the cell and core, which are gathered in autophagosomes. While osmotic anxieties cause autophagy using the objective of rapamycin target of rapamycin kinase pathway, oxidative and ER stress conditions lead to autophagy through the inositol-requiring catalyst 1 (IRE1) pathway. Certain food varieties that cause ER stress and diseases (such as flu and COVID) start the aggravation course and advance viral replication by speeding up the cycle of autophagy. COVID initiates the development of layer vesicles to which viral replication and record buildings are related. These layer vesicles lead to ER stress interceded autophagy [7]

Even though there is no proof that SARS-Cov-2 causes ER stress-intervened autophagy, Fung et al. showed that viral replication could be diminished by hindering the IRE1 pathway in COVID contaminated patients with bronchitis. In such as manner, comparable to autophagy, Kong et al. announced that interleukin (IL)-6 might increment autophagy using the IRE1 pathway. IL-17 (starting fundamentally from IL-17delivering $\mathrm{T}$ cells) is supportive of incendiary and carcinogenic cytokine that assumes a significant part in the versatile insusceptible framework. IL-17 is likewise an intense inducer of ER stress and autophagy utilizing IRE1. As needs are, an IL-17 barricade has been displayed to forestall ER stress-related autophagy and inflammation. IL-17 additionally assumes a significant part in incendiary inside sickness. Since impacts of this novel COVID on the gastrointestinal (GI) tract have been very much recorded, it is sensible to recommend that these impacts may intercede through IL-17. In addition, Hou et al. showed that the presence 
of over-the-top IL-6 advances the age of Th17 cells and that IL-6 and IL-17 synergistically advance viral replication and might be significant focuses for hostile to COVID treatments. Corneth et al. exhibited that a shortfall of the IL-17 receptor prompts a decline in IL-6 creation. Notwithstanding, Colaneri et al. revealed that contradicting effect of IL- 6 treatment alone neglected to decrease concentrated consideration affirmation rates and mortality in CoV patients [1]. Competent immune system is important in eliminating SARS-CoV-2 from host. Zink plays an important role in maintaining immune function both in innate and adaptive immune system. Zink is also suggested as a therapy for COVID-19 cases [12].

\section{Possible Link between Gut and Lungs Microbiomes and their Modulation}

The term microbiome incorporates the whole microbial local area such as microorganisms, archaea, parasites, and infections. Advances in research have prompted the agreement that there is a powerful crosstalk between the organisms of the gut-lung pivot. This advancement shuddered the old authoritative opinion of the sterile lung climate. Connecting of the gut and the lung specialty intercedes through this pivot as it is a course for the section of chemicals, microbial metabolites, cytokines, and endotoxins into the circulation system. A reasonable gut local area is of imperative significance in aspiratory invulnerability. A few investigations recommend that dysbiosis in gut microbiota impact aspiratory brokenness by balancing the safe reactions of neutrophils, $\mathrm{T}$ cell subsets, provocative cytokines, for example, Toll-like receptors (TLR), and some more. The neighborhood or distal resistant adjustment of the commensal organisms in the lungs and gut influences the beginning of the disease cycle. Nonetheless, the native gut commensals give colonization opposition from the microbial microorganisms by the idea of "obstruction impact" and hence, help in shielding the gut specialty from being changed [13].

During the disease of the respiratory tract, the commensal organic entities of our body animate the neighborhood (from lungs) and the adjoining distal resistant reaction (at the locales of the gut). The gutlung pivot is thought to be bidirectional, which means disease by SARS-CoV-2 at the lungs triggers a safe reaction in the $\mathrm{Gl}$ tract. The disease of the lungs with SARS-CoV-2 causes an epithelial interruption in the gas trade regions and the related aviation routes. Epithelial cells of the alveoli with angiotensin-converting enzyme 2 (ACE2) receptor fill in as the limiting site for SARS and SARS-CoV-2. The grouping of favorable to fiery cytokines (Interferon [IFN] $\gamma$-instigated protein 10; monocyte chemoattractant protein 1 and $\mathrm{IL}-8)$.
Overproduction of cytokines and chemokines, actuation of $\mathrm{T}$ assistant cell-interceded invulnerability, and host fiery reaction were seen during the intense period of SARS and SARS-CoV-2 contamination. Ongoing investigations propose that the association of the gut in COVID-19 is significantly more prominent and more drawn out contrasted and the lung. Strikingly, it has been accounted for that probiotics show huge microbial inhibitory properties through alveolar macrophage, neutrophils, normal executioner cells, and expanded degrees of favorable to incendiary cytokines such as tumor necrosis factor $\alpha$ (TNF- $\alpha)$ and IL-6 in the lung. Furthermore, probiotic microbes can tie the attacking infection and repress the microorganism have cell receptor association. Consequently, the utilization of probiotics as a drug confines respiratory viral diseases by sustaining mucosal insusceptibility [8], [14].

Probiotic microorganisms have been displayed to have various helpful safe and wellbeing impacts. They did not just upgrade the bioavailability of supplements and moderate wellbeing, and they are likewise associated with directing the bacterial biological system and module invulnerable cells. Dendritic cells (DC) assume a critical part in safe homeostasis in the solid digestive system. DCs are key antigen introducing cells that take up antigens (for example, viral and malignant growth) and present little antigenic peptides on their surface to prime T cells toward pro-inflammatory (Th1) or calming (Th2) aggregates. DC in a youthful state can prompt erasure of T cells or incitement of administrative T cells. The gut microbiota can drive DC to prime these cells. Indeed, L. reuteri and L. casei, invigorate IFN- $\gamma$ creation and actuate favorable to fiery Th1 cells [13].

Moreover, oral organization of $B$. infant is in mice animate DCs which smother Th2-one-sided reactions and invigorate Th1 proinflammatory reactions which are needed for infection end. Monocytes are available in the fringe blood which is among the primary cells to be in touch with microbes and infections. They separate into tissue macrophages, where intestinal microbiota or ingested probiotics associate with them to discharge various cytokines. The supportive of incendiary cytokine, IL-12 is emitted by macrophages which invigorate normal executioner cells and CD4+ Th1 cells to discharge IFN- $\gamma$ which are needed for disposal of infections [15]. Furthermore, probiotics Lactobacillus gasseri, Lactobacillus delbrueckii ssp. bulgaricus, Bifidobacterium bifidum, Lactobacillus acidophilus strains instigate IFN- $\alpha$ creation by monocytes. The probiotic Lacticaseibacillus paracasei DG expands TNF- $\alpha$, IL-6, IL- 8 of the human monocyte cell line, THP-161. In addition, it was as of late noticed that Streptococcus thermophilus instigated TNF- $\alpha$, IL-6, IL-8 profile which is needed against viral impacts [16].

Natural killer (NK) cells are significant in the early invulnerable reaction against viral diseases, specifically through the leeway of infectioncontaminated cells. Lactobacillus probiotic strains can 
invigorate DCs to emit IL-12, which thusly initiates NK cells to discharge IFN- $\gamma$, a fundamental cytokine for lung bacterial (Staphylococcus aureus) and viral end. Probiotics such as Lacticaseibacillus casei can likewise interface with TLR on the epithelial cells, in this manner, upgrading the creation of cytokines that assume a significant part in further developing the usefulness of the epithelial cell and forestalling their apoptosis which improves their endurance and multiplication during reclamation. Understanding the invulnerable cell enactment, cytokine profiles and safe adjustment is significantly giving a make way to overseeing viral diseases [6], [8].

\section{Therapeutic Implications}

These discoveries recommend that a procedure that consolidates a more exhaustive way to deal with safe adjustment as opposed to the hindrance of individual cytokines might be more viable against the cytokine storm in infection-tainted patients. IL-17 and IL-6 advance viral diligence by safe collaborations through autophagy. Schett et al. detailed that impeding IL-17 may decrease viral replication in COVID-19 patients. The pathogenesis of the resistant reaction to COVID looks similar to TH17-Th1 driven immune system illnesses and these TH17-Th1 insusceptible communications seem to assume a significant part in infection replication [13].

The resistant regulating impacts of probiotics incorporate against the IL-17 impact which could demonstrate significance in both treatment and immunization improvement. Besides, invulnerable connections, through autophagy, will influence coronaviral replication. In this unique circumstance, probiotics may assume a significant part in the administration of COVID-19. Late proof has shown that the impact of the SARS-CoV-2 infection isn't restricted exclusively to the respiratory parcel. The SARS-CoV-2 infection has been recognized in the digestion tracts of COVID-19 patients and looseness of the bowels is one of the introducing indications in 2-half of the recently conceded COVID-19 patients [14].

The utilization of probiotics for the treatment of COVID-19 patients is a functioning space of clinical exploration. Of 13 preliminaries enlisted with ClinicalTrials.gov, just one has been finished to date, yet the outcomes have not been distributed. Just one investigation has been distributed to date utilizing probiotics for the treatment of COVID-19 patients. D'Ettorre et al. selected 70 patients with stage III COVID infection conceded to an emergency clinic in Rome, Italy $(94 \%$ had a fever and $47 \%$ had the runs at affirmation). All patients were treated with standard treatments (hydroxychloroquine, azithromycin, or potentially tocilizumab) and afterward arbitrarily picked patients were additionally given an eight-strain probiotic blend. Essentially more patients with loose bowels on affirmation who took the probiotic blend had their runs settled by day $3(93 \%)$ contrasted with patients who didn't take the probiotic combination $(5 \%, p<0.001)$. Strangely, the danger of respiratory disappointment was additionally altogether higher for those not taking the probiotic blend (Odds Ratio = 8.6, 95\% C.I. 1.6-45.0). Regardless of whether probiotics will demonstrate accommodating in treating COVID-19 infection and difficulties relies on the aftereffects of continuous clinical preliminaries [13], [14].

Given the key significance of gut microbiota in impacting lung sicknesses, the designated control of gut microorganisms utilizing certain dietary enhancements proposes a promising helpful methodology. Arising contemplates recommending the utilization of probiotic microscopic organisms in the therapy or avoidance of a wide scope of human infections, ailments, and disorders. Probiotics are live microorganisms that, when controlled insufficient sums, give a medical advantage to the host. Probiotic systems in preventive and restorative methodologies comprise of changing the intestinal microbial networks, immunomodulation, clipping down the microorganisms, and assurance of the intestinal hindrance. They have effectively been utilized in the therapy of anti-infection-related runs, incendiary gut illness, and diverse ongoing provocative sicknesses. Insusceptible framework balance is a critical factor in the anticipation of irresistible illnesses. Probiotic microorganisms have exhibited their capacity to animate and balance the invulnerable framework and to decrease aggravation [17].

Probiotics are known to diminish the seriousness of contaminations in the GI tract and the upper respiratory tract by following up on both the natural and the versatile resistant frameworks. At present, the utilization of probiotic microorganisms and their metabolic items addresses a promising methodology for the treatment of viral sicknesses. Colonization of intestinal epithelium by the probiotic microorganisms has been displayed to diminish the rates and indications of viral respiratory contaminations. This is accomplished by the upsurge of Immunoglobulin A communicating $B$ cells in the colon and lymph hubs related to the expanding populace of the $\mathrm{T}$ follicular partner cells and IL-23-communicating DC. Besides, probiotics likewise involve immunostimulatory constituents, for example, peptidoglycan, lipoteichoic corrosive, TLR ligands, and muramyl dipeptide, which emphasize their immunomodulatory power. The new investigation of $\mathrm{Ji}$ et al. showed that the supplementation of probiotics to respiratory syncytial virus-contaminated mice has essentially raised the bounty of short-chain unsaturated fat (SCFA) delivering gut microbiota which thusly up-direct the creation of IFN- $\beta$. Furthermore, they have additionally revealed the upsurge of Corynebacterium 
and Lactobacillus species in the lung due to higher SCFA creation, subsequently prompting the actuation of interferon $\beta$ creation in alveolar macrophages [18].

A randomized, two-fold visually impaired and fake treatment controlled human investigation of 109 grown-ups showed the upgraded level of mitigating cytokines IL-4 and IL-10 and decreased plasma peroxidation and an oxidative endless supply of Lactiplantibacillus plantarum DR7. The COVID-19 contamination influences the lung tissues and gut, consequently enacting the fiery reaction. It expands the proinflammatory cytokines (IFN- $\gamma, \mathrm{TNF}-\alpha)$ which lead to the rise of the cytokine storm. This reaction is presumably a result of the initiation of $T$ partner cells (Th1) cell reaction in the lung tissue. On account of the human gut climate, dysbiosis in the gut microbiota brings about the unevenness of Th1 and Th2 which further outcomes in the actuation of proinflammatory cytokine and at last the cytokine storm in the lungs also. Endless supply of probiotics, there is the colonization of supposed "great microorganisms" in the gut which prompts a change yet to be determined between Th1/Th2 cells that diminishes the cytokine storm and decreases the seriousness of illnesses [13].

As of late, it has been discovered that drug with probiotic microscopic organisms utilizing Bifidobacteria and Lactobacillus gives a critical possibility of recuperation against COVID-19. Beforehand, these probiotic microscopic organisms Probiotics were accounted for to have helpful impacts against respiratory contamination by the flu infection. The organization and utilization of probiotics advance the safe framework by improving the degree of type I interferons, antigenpresenting cells, NK cells, and B and T cells of the lungs. Probiotic organizations can likewise work on the supportive of and calming cytokines, assisting with clearing the viral disease by limiting the phone harm in the lungs [14].

\section{Probiotics Role within Antiviral Activity}

Probiotics can handle drug-related looseness of the bowels and $\mathrm{Gl}$ tract contamination, and control different locales of diseases such as respiratory tract contaminations and sepsis. For proof-based medications, the best quality level would be metainvestigation. In one examination on roughly 9000 babies included in randomized controlled preliminaries, the mother's admission of probiotics through the GI tract could diminish necrotizing enterocolitis, nosocomial sepsis, and mortality hazard. An efficient randomized controlled preliminary in India comprising of more than 5,000 babies treated with Lactobacillus plantarum strain related with prebiotics showed a declining level of sepsis and lower respiratory tract contaminations. Infections are the primary factor causing upper respiratory tract contaminations. In the counteraction of upper respiratory tract diseases, the useful impact of probiotics has been affirmed. Different investigations of 13 randomized controlled preliminaries have been directed which contains 4230 young people and children who were given probiotics; there was a 2-overlay decline in hazard of lower and upper respiratory tract contaminations and a lower yet vital lessening in the seriousness of illness in the transporter patients. Besides, there was a randomized, twofold dazed fake treatment controlled examination with 523 young people who were given L. gasseri PA 16/8, Bifidobacterium longum SP 07/3, and B. bifidum MF $20 / 5$, for certain minerals and nutrients. The outcomes showed lower influenza periods and a decrease in fever days [18].

A randomized controlled preliminary containing 93 babies was given galacto-oligosaccharides and polydextrose prebiotic mix (1:1) and Lacticaseibacillus rhamnosus GG in 3-60 days. Treatment diminished by 2-to 3-overlay the event of respiratory tract viral diseases when contrasted with the fake treatment control. The event of rhinovirus-related occasions which incorporate $85 \%$ of all respiratory parcel contamination cases in this investigation was likewise exceptionally diminished with the utilization of prebiotics and probiotics. Lactobacillus brevis has additionally shown promising outcomes in an investigation of 1692 school kids and emphatically decreased the event of flu respiratory parcel diseases. In examinations, the flare-up has troubled the youths than kids, yet $B$. longum has been reported in a randomized controlled preliminary of 28 grown-ups and has likewise shown promising outcomes. Besides, numerous probiotics are being chosen from lactic corrosive microbes that are generally found in the upper respiratory tract of sound people, and the vast majority of the probiotic strains are recorded in the counteraction of steady otitis media. Consequently, it tends to be reasoned that probiotics have a legitimate commitment in hindering the occurrences of COVID episodes [13].

Aside from use in viral diseases, probiotics can likewise help in decreasing bacterial contaminations such as lower respiratory tract diseases in grownups. Numerous test information has been gotten from a randomized controlled preliminary containing 2000 contaminated patients of VAP. In this examination, the outcomes from more than 1800 grown-ups showed recuperation and a decrease of episodes. Be that as it may, proof of lower quality and conflicting outcomes in different preliminaries warrants further examinations with an efficient randomized and controlled plan. It should be noticed that not all probiotics are implied in diminishing the danger of respiratory tract diseases, even the probiotics with $\mathrm{Gl}$ advantages. For instance, Bifidobacterium animalis and L. rhamnosus may add to the intestinal guide however don't diminish the infection number in the nasopharynx [8], [14]. 
Lactic acid bacteria are known to create an assortment of antimicrobial substances such as acids, peptides or proteins, non-ribosomal peptides (NRP), hydrogen peroxide, and different metabolites. Hydrogen peroxide is poisonous to numerous non-catalase microorganisms; be that as it may, their enemy of respiratory tract viral action isn't known yet their action against human immunodeficiency infection HIV-1 and Herpes simplex infection herpes simplex virus type 2 (HSV-2) were accounted for before. Lactic corrosive, the result of carb digestion is a significant microbicidal compound, it kills corrosive delicate organisms. It helps the host cells in forestalling viral replication. Besides, the articulation and ACE-2, a receptor needed by COVID-19 infection for its limiting is displayed in L. paracasei. Restricting this emitted ACE-2 with COVID-19 restricting protein can forestall its entrance into the cell and hence decreased the odds of contamination [19].

Antimicrobial peptides are created by probiotics life forms are the particles most described for their antimicrobial action and hostile to the viral movement. Bacteriocins are the antimicrobial peptides created by genera Lactobacillus and Enterococcus spp. having a wide range of action against different Gram-positive and Gram-negative microorganisms. It very well may be utilized as options in contrast to anti-toxins or blend with anti-toxins. Bacteriocin mixtures, for example, staphylococcin 188, enterocin AAR-74, erwiniocin NA4 have been assessed for antiviral action. Their movement is accounted for against HIV, HSV, Coliphage, flu infection, and especially $\mathrm{H} 1 \mathrm{~N} 1$ infection. Additionally, the NRPs are likewise the auxiliary metabolites created by probiotic microorganisms that have exceptionally expansive clinical applications. Their utilizations are accounted for as anti-infection agents (daptomycin), against tumor drugs (bleomycin), antifungal medications, and immunosuppressants (cyclosporin) [19], [20].

Studies that stated about the accomplishment of probiotics in clinical preliminaries have demonstrated boundaries such as improvement of the epithelial hindrance of digestion tracts, contest with infection-causing specialists for supplements, connection on the epithelium mass of digestive organs, creation of antipathogen components, and upgrade of the invulnerable arrangement of the host [21]. A randomized controlled examination plan of 63 infants who got enteral sustenance alongside $B$. bifidum and $S$. thermophilus showed a decrease in diarrheal occurrences and decrease of rotavirus shedding, as shown by results acquired from the meta-investigation. These discoveries demonstrate a break in the passage of the infection into the cell and restraint of viral replication in the host digestive tract. Along these lines, this framework might add to the decrease of COVID intrusion through the $\mathrm{GI}$ tract since probiotics are not taken in through the respiratory tract [14].

Thusly, at this site, direct counteraction of infections probably won't be conceivable. It is likewise revealed that the microbiota is additionally found in the lungs; gut-lung affiliation has been talked about whereby microorganism to-organism and host-to-organism resistant joint efforts may invigorate the movement of respiratory diseases. Flu-type diseases are identified with the disparity of microbial gatherings of the $\mathrm{Gl}$ and respiratory parcels [22]. This dysbacteriosis may change the elements of the insusceptible framework and brief optional bacterial contamination. As studies from China have shown, COVID-19 might be going with intestinal dysbacteriosis, in this manner causing extreme contamination; reclamation of gut homeostasis might be helped through probiotic strains. It is sensible that probiotic strains, when orally controlled, could invigorate gut-lung affiliation and a few strains can relocate from the gut to other closer locales, similar to the bosom, to cure mastitis [22].

The microbiota of the gut significantly affects the host insusceptible reaction, foundationally, and on invulnerable responses at close by mucosal locales, such as the lung. Utilization of specific bifidobacterial and lactobacilli strains can positively affect the progression of flu infection from the respiratory tract. Certain strains of probiotics have improved interferon type I levels, hoisting the number and capacity of antigen-introducing cells, regular executioner cells, and $T$ cells, just as expanding the degree of specific antibodies at the fundamental and mucosal destinations. There is likewise verification that strains of probiotics can revise the security among favorable to provocative and immunoregulatory cytokines that grant the freedom of infections. This could be generally fitting to deflect the intense respiratory trouble condition, a significant obstacle of COVID-19 [20], [23].

A randomized controlled plan incorporates young people who show concealment of plasma favorable to fiery cytokines, for example, IFN-g and TNF-a, with the utilization of Lactobacillus DR7. Another preliminary of youthful grown-ups showed improvement in mitigating cytokines, similar to IL-4 and IL-10, and a decrease in the peroxidation of plasma cells and oxidative feelings of anxiety. Certain tempests of cytokines may create in patients with COVID-19, and this tweak type may end up being exceptionally critical. The strategy wherein strains of probiotic are orally devoured would add to the rise of the safe reaction from the digestive organs, a pivotal place of the host protections. Thusly, strains of probiotics are known to work on the immovability of tight intersections; for example, they could go about as fuel for colonocytes which could probably decrease the intrusion of SARSCoV-2 by expanding the degree of butyrate. Probiotic strains have additionally been exhibited to apply hostile to the viral movement, as displayed in trial and clinical studies [24].

Nonetheless, none of these systems and impacts have been confirmed on SARS-CoV-2. This methodology ought not to be declined, especially when probiotic strains have been decidedly tried against different strains of COVID. In addition, patients are likewise as of now 
influenced by auxiliary bacterial contaminations [25]. An earlier report showed that mice with aspiratory disease because of Pseudomonas aeruginosa and S. aureus, when orally controlled with probiotic strains of $L$. acidophilus, exhibited a decline in the bacterial weight from the lungs and a decrease in the odds of lung disability and foundational contaminations [18].

\section{Probiotics Safety Consideration as Prevention and Management of COVID-19}

Probiotics are typically innocuous even in the most helpless networks and clinic settings. A few cases with bacteremia and fungemia identified with probiotics have emerged for the most part in immunocompromised people, because of the shortfall of proper quality control; in any case, these have happened in uncommon circumstances. More often than not, the probiotics are innocuous, yet it relies on the portion of probiotics. If we utilize the legitimate portion of probiotics, we can get the most extreme outcomes to control any disease, here, for this situation, to forestall the viral contamination. Be that as it may, assuming the portion of the probiotics is high; it might cause a few complexities [26].

Besides, in a randomized controlled investigation of 72 basic patients comprising of different injury guardians and instinctually ventilated people, the organization of Pediococcus pentosaceus, Leuconostoc mesenteroides, L. paracasei, L. plantarum, insulin, oat wheat, gelatin, and starch resulted in abatement in the pace of irritation, foundational contamination reaction illness, sepsis, and length of stay in medical clinics, particularly on ventilators, just as a reduction in death rate [27].

The role of probiotics in respiratory viral infection, including COVID-19, can be seen in Figure 1.

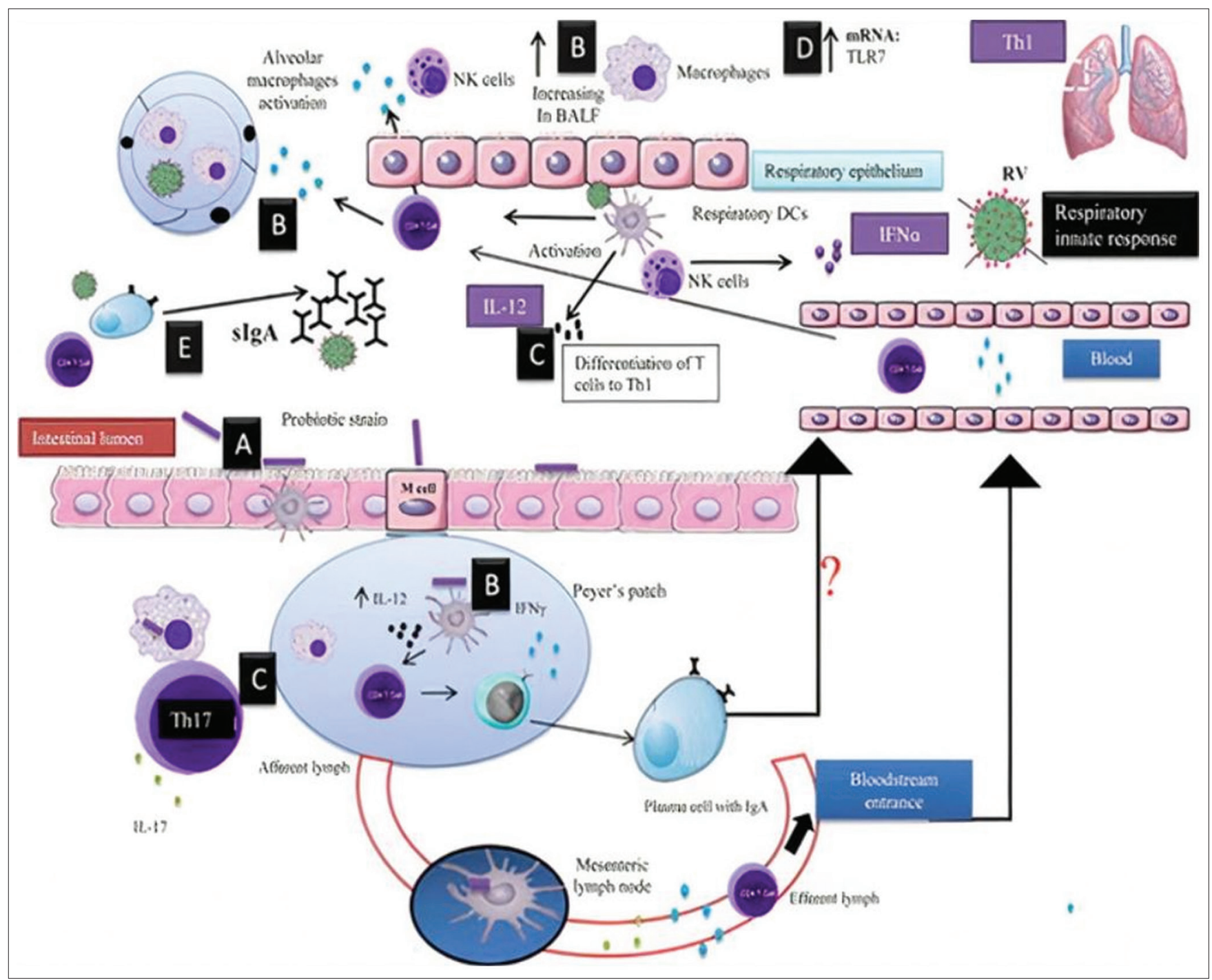

Figure 1: The role of probiotics in the pathogenesis of respiratory viral infection. (A) Probiotics interact with intestinal epithelium and stimulate Dendritic cells to produce Interferon $\gamma$ and interleukin-12. (B) Both pro-inflammatory cytokines disseminate to systemic circulation and aid alveolar macrophages and natural killer cells to eliminate virus. (C) Probiotics increase the production of Th1/Th17 and increase CD4+ and CD8+ which important in innate immune system. (D) Lung toll-like receptors 7 expression is also increased which support viral elimination. (E) Lastly, probiotics stimulate the differentiation of plasma cells systemically to produce immunoglobulin A [18] 
Diet, Probiotic, and COVID-19

Diet rich in probiotics and prebiotics is suggested to have positive impact toward COVID-19. Diary fermentation products such as yogurt, kefir, buttermilk, cheese, cheddar, and mozzarella possess abundant lactobacilli which releases highly-affinity peptides for ACE2 [28], [29]. Adequate amount of prebiotics may also enhance the role of probiotics in modulating immune system. Prebiotics act as important nutrition source for the growth of probiotics. Examples of prebiotic source are Chinese yam, whole wheat, barley, brown brown rice, legumes, mushrooms, lentils, and nuts. Furthermore, high intake of animal proteins, saturated fat, sugar, ans salt has negative impact toward probiotics. During this pandemic, diversified diet with moderate increase in high-fiber and plant-based foods is advised such as Mediterranean diet [28].

\section{Conclusion}

Probiotics have various advantages, for example, adjusting the arrangement of human gut microflora, reinforcing gut obstruction work, and defensive invulnerable reactions. Numerous viral diseases are related to dysbiosis of gut microbiota prompting extreme GI tract contaminations. Probiotics can tweak have invulnerable reactions and neutralize the "cytokine storm" delivered during COVID-19 contamination. Notwithstanding, utilizing a probioticsbased procedure against COVID-19 disease in clinical and restorative fields is as yet an open exploration question. Step by step instructions to choose standard bacterial species for a populace, since different confounded elements, such as age, way of life, dietary propensities, and hereditary qualities, impact the human gut microbiota.

\section{References}

1. Khaled JM. Probiotics, prebiotics, and COVID-19 infection: A review article. Saudi J Biol Sci. 2021;28(1):865-9. https://doi. org/10.1016/j.sjbs.2020.11.025

PMid:33424377

2. Ruslie RH, Darmadi D. Coronavirus disease-19 and mental health. Open Access Maced J Med Sci. 2020;8:268-71. https:// doi.org/10.3889/oamjms.2020.5304

3. Siregar GA, Siregar GP, Darmadi D, Ruslie RH. Coronavirus Disease-19 and Liver Injury. Open Access Maced J Med Sci. 2020;8:154-7. https://doi.org/10.3889/oamjms.2020.5028

4. Mustafa SK, Ahmad MA, Baranova V, Deineko Z, Lyashenko V, Oyouni AA. Using wavelet analysis to assess the impact of
COVID-19 on changes in the price of basic energy resources. Int J Emerg Trends Eng Res. 2020;8:2907-12. https://doi. org/10.30534/ijeter/2020/04872020

5. Telles RC, Roy A, Ajmal MR, Mustafa SK, Ahmad MA, de la Serna JM, et al. The impact of COVID-19 management policies tailored to airborne SARS-CoV-2 transmission: policy analysis. JMIR Public Health Surveill. 2021;7:e20699. https://doi. org/10.2196/20699

\section{PMid:33729168}

6. Bhushan I, Sharma M, Mehta M, Badyal S, Sharma V Bioactive compounds and probiotics-a ray of hope in COVID19 management. Food Sci Hum Wellness. 2021;10(2):131-40. https://doi.org/10.1016/j.fshw.2021.02.001

7. Kurniawati EM, Rahmawati NA, Surgeanveterini A. Considering role of probiotic on respiratory disease: Is probiotic possible to treat COVID-19 ? Int J Forensic Med Toxicol. 2021;15(2):40038. https://doi.org/10.37506/ijfmt.v15i2.14998

8. Bozkurt HS. The probiotic Bifidobacterium in the management of coronavirus: A theoretical basis. Int $\mathrm{J}$ Immunopathol Pharmacol. 2020;34:2058738420961304. https://doi. org/10.1177/2058738420961304

PMid:33103512

9. Siregar GA, Siregar GP, Darmadi D. Gastrointestinal aspects of COVID-19: A review. Open Access Maced J Med Sci. 2020;8:524. https://doi.org/10.3889/oamjms.2020.4891

10. Ruslie RH, Darmadi D, Siregar GA. Pediatric immunization practice during coronavirus disease-2019 pandemic. Open Access Maced J Med Sci. 2020;8:258-60. https://doi. org/10.3889/oamjms.2020.5125

11. Ruslie RH, Darmadi D, Siregar GA. Susceptibility of coronavirus disease-19 in pediatric population. Open Access Maced J Med Sci. 2020;8:363-5. https://doi.org/10.3889/oamjms.2020.5240

12. Mustafa SK, Aljohani MM, Alomrani NA, Oyouni AA, Alzahrani O, Ahmad MA, et al. COVID-19 an immune function a significant zinc. Orient J Chem. 2020;36:1026-36. http://dx.doi. org/10.13005/ojc/360604

13. Kullar R, Johnson S, McFarland L. Potential roles for probiotics in the treatment of COVID-19 patients and prevention of complications associated with increased antibiotic use. Antibiotics (Basel). 2021;10(4):408. http://doi.org/10.3390/ antibiotics10040408 PMid:33918619

14. Gohil K, Samson R, Dastager S, Dharne M. Probiotics in the prophylaxis of COVID-19: something is better than nothing probiotics in the prophylaxis of COVID-19: Something is better than nothing. 3 Biotech. 2020;11:1-12. https://doi.org/10.1007/ s13205-020-02554-1

15. Darmadi D, Ruslie RH. Association between serum interleukin (IL)-12 level and severity of non-alcoholic fatty liver disease (NAFLD). Rom J Intern Med. 2021;59(1):66-72. https://doi. org/10.2478/rjim-2020-0029 PMid:33055315

16. Ruslie RH, Tjipta DG, Samosir CT, Hasibuan BS. Bacterial pattern and role of laboratory parameters as marker for neonatal sepsis. IOP Conf Ser Earth and Environ Sci. 2018;125(1):012057. https://doi.org/10.1088/1755-1315/125/1/012057

17. Belojevic $\mathrm{G}$. Comparative analysis of vaccines and probiotics in fighting the Covid19. Biomed J Sci Tech Res. 2021;1:26366-8. https://doi.org/10.26717/BJSTR.2021.34.005487

18. Zafar N, Aslam MA, Ali A, Khatoon A, Nazir A, Tanveer Q, et al. Probiotics: Helpful for the prevention of COVID-19? Biomed Res Ther. 2020;7(11):4086-99. https://doi.org/10.15419/ bmrat.v7i11.646

19. Ceccarelli G, Scagnolari C, Pugliese F, Mastroianni CM, d'Ettorre G. Probiotics and COVID-19. Lancet Gastroenterol Hepatol. 2020;5(8):721-2. https://doi.org/10.1016/ 


\section{S2468-1253(20)30196-5 PMid:32673604}

20. Akour A. Probiotics and COVID-19: Is there any link? Lett App Microbiol. 2020;71(3):229-34. https://doi.org/10.1111/lam.13334 PMid:32495940

21. Zendeboodi F, Khorshidian N, Mortazavian AM, Cruz AG. Probiotic: conceptualization from a new approach. Curr Opin Food Sci. 2020;32:103-23. https://doi.org/10.1016/j. cofs.2020.03.009

22. Olaimat AN, Aolymat I, Al-Holy M, Ayyash M, Ghoush MA, Osaili T, et al. The potential application of probiotics and prebiotics for the prevention and treatment of COVID-19. npj Sci Food. 2020;12:1-6. https://doi.org/10.1038/s41538-020-00078-9

23. Darbandi A, Asadi A, Ghanavati R, Roghayeh A, Darb A, Talebi M. The effect of probiotics on respiratory tract infection with special emphasis on COVID-19: Systemic review 201020. Int J Infect Dis. 2021;105:91-104. https://doi.org/10.1016/j. ijid.2021.02.011 PMid:33578007

24. Rozga M, Cheng FW, Handu D. Effects of probiotics in conditions or infections similar to COVID-19 on health outcomes: An evidence analysis center scoping review. J Acad Nutr Diet. 2020;121(9):1841-54. https://doi.org/10.1016/j. jand.2020.07.016

PMid:32807723

25. Singh K, Rao A. Probiotics: A potential immunomodulator in COVID-19 infection management. Nutr Res. 2021;87:1-12. https://doi.org/10.1016/j.nutres.2020.12.014

26. Mak JW, Chan FK, Ng SC. Probiotics and COVID-19: One size does not fit all. Lancet Gastroenterol Hepatol. 2020;5(7):644-5. https://doi.org/10.1016/S2468-1253(20)30122-9

PMid:32339473

27. Bottari B, Castellone V, Neviani E. Probiotics and Covid-19. Int J Food Sci Nutr. 2021;72(3):293-9. https://doi.org/10.1080/0963 7486.2020 .1807475

28. Hu J, Zhang L, Lin W, Tang W, Chan FK, Ng SC. Probiotics, prebiotics and dietary approaches during COVID-19 pandemic Trends Food Sci Technol. 2021;108:187-96. https://dx.doi. org/10.1016\%2Fj.tifs.2020.12.009

PMid:33519087

29. Mirashrafi S, Moravejolahkami AR, Zehi ZB, Kermani MA, Bahreini-Esfahani $\mathrm{N}$, Haratian $\mathrm{M}$, et al. The efficacy of probiotics on virus titres and antibody production in virus diseases: A systematic review on recent evidence for COVID19 treatment. Clin Nutr ESPEN. 2021. https://doi.org/10.1016/j. clnesp.2021.10.016 